\title{
Development of SphagneticolaTrilobata Flowers Extract Coated Urinary \\ Catheters: Determining Its Antibacterial Activity against Escherichia coli and Staphylococcus aureus
}

\author{
Bhavadharani ${ }^{1}$, Reshmi Gopalakrishnan ${ }^{1}$, Karkuvelraja $\mathbf{~ R}^{2 *}$
}

\begin{abstract}
Author details
${ }^{1}$ Department of Microbiology, Sri Ramakrishna College of Arts and Science for Women, Coimbatore, TamilNadu-641044, India

${ }^{2}$ Microbial Biodiversity and Bioenergy Lab, Department of Microbiology, Bharathidasan University, Tiruchirapalli-620024, India(*Corresponding author)
\end{abstract}




\begin{abstract}
:
Urinary catheters are regularly used within side the elderly for each short- or long-term. The biofilm formation occurs on the catheters and leads to urinary tract infection. The present study focus on development of Sphagneticolatrilobata coated catheters for prevention of urinary tract infections (UTI). Bioactive compounds were extracted using methanol and MIC was determined. Biofilm inhibition assay was determined by Fluorescent microscopic analysis. $7.5 \mathrm{mg} / \mathrm{ml}$ extracts showed $13 \mathrm{~mm}$ against $E$. coli and $14 \mathrm{~mm}$ against $S$. aureus. The extract coated catheter showed significant reduction in biofilms from fluorescent microscopic analysis. Hence Sphagneticolatrilobataflower extract coated catheters can be used to prevent biofilm colonization and urinary tract infections.
\end{abstract}

Keywords:Sphagneticolatrilobata, Urinary catheters, biofilm assay, Fluorescent microscope 


\section{INTRODUCTION:}

A biofilm is a collection of cells developing on a floor and enclosed in an exopolysaccharide matrix. Biofilms are notoriously hard to eliminate and are a supply of many recalcitrant infections. (Kim Lewis, 2001). Biofilm formation is a key step withinside the pathogenesis of many subacute and persistent bacterial infections (which includes overseas bodies. Traditional antibacterial sellers are hard to get rid of biofilms. Bacterial biofilms have numerous capacity resistance mechanisms. Antimicrobial resistance mechanisms can paintings simultaneously, and in a few instances can paintings synergistically. Persistent cells play a prime position withinside the resistance of biofilm micro organism to antibacterial dealers. (Robin Patel and JL Del Pozo,2007) .

Urinary catheters are regularly used withinside the elderly for each short- or long-term catheters, the infection rate is prepared 5\% constant with day. Escherichia coliremain the most now no longer unusualplace infecting organism, but a large kind of unique organisms may be isolated, which incorporates yeast species. Bacteria typically have a tendency to show increased resistance because of the repeated antimicrobial courses. Urinary tract infection (UTI) commonly follows formation of biofilm on every the internal and out of doors catheter floor..The biofilm protects organisms from every antimicrobials and the host immune response. Morbidity from UTI with short-term catheter use is restrained if appropriate catheter care is practised. In patients with long-term catheters, fever from a urinary deliver isn't always unusualplace with a frequency diverse from 1 consistent with 100 to at the least one constant with 1000 catheter days. Longterm care facility residents with continual indwelling catheters have a much more chance for bacteraemia and exclusive urinary complications than residents without catheters (Lindsay E Nicolle., 2005) .

Biofilms are answerable for numerous infectious diseases. Some traditional examples are: dental caries (as a result of acidogenic Gram-advantageous cocci Streptococcus sp.), periodontitis(Gram-terrible anaerobic oral microorganism), otitis media, or center ear infection (non-typeableHaemophilus influenza), continual tonsillitis (with the aid of using diverse species), cystic fibrosis pneumonia (Pseudomonas aeruginosa, Burkholderiacepacia), endocarditis (streptococci, staphylococci), necrotizing fasciitis (Group A streptococci), musculoskeletal infections (Gram-advantageous cocci), osteomyelitis (numerous species), biliary tract infection 
(enteric micro organism), infectious kidney stones (Gram-poor rods), bacterial prostatitis (Escherichia coli and different Gram-poor micro organism). Organisms like P. aeruginosa, Staphylococci and E. coli are related to infections due to overseas frame substances, including infection of implants, catheters, touch lenses and prostheses(Fux CA et al., 2005).

Reliable vascular access is an important function of current day health care and for decades biomaterials have achieved an crucial function in illness control (Bazaka K et al.,2011). Among biomaterials, the most extensively employed are catheters - smooth tubing for the delivery of substances over time, implanted from the out of doors ground into the organ or vessel of hobby.Intravascular catheters are used particularly withinside the control of fluids, medications, parenteral nutrition, and blood products, further to withinside the monitoring of hemodynamic reputation and in chronic outpatient hemodialysis (Mermel LA et al.,2011).Unfortunately, the usage of devices for vascular access is associated with an underappreciated threat of intravascular device-related bloodstream infection, because of microorganisms that colonize the implanted device or contaminate the fluid Pathway, at the time of insertion or withinside the direction of its use (Maki DG et al.,2006). Two barriers are violated all through vascular catheterization: the pores and pores and skin and the vein wall.This lets in the contact of the encompassing carefully inflamed with the blood go with the flow alongside interfaces: the outer ground of the catheter and the catheter lumen (Girvent M. et al., 1999).

Prevention of catheter-received UTI and its complications is a major goal. With quickterm catheters, warding off their use or limiting the period of use to as short a time as possible are the best prevention techniques. Maintaining a closed drainage tool and adhering to appropriate catheter care techniques will also limit infection and complications. As the duration of catheterisation is the primary determinant of infection with long-term indwelling catheters (Nicolle., 2005)

Sphagneticolatrilobata is an invasive herb in South China. Here, we suggested a few morphological intermediates among $S$. trilobata and the indigenous congener $S$. calendulaceawithinside the discipline for the primary time, which have been suspected to be hybrids.(HaoShenet al.,2013). S. trilobatais a member of own circle of relatives Asteraceae that has been applied as antique conventional medication in India, Chinese, Caribbean, and Central 
and South America. It is thought as "Wedelia" withinside the Pacific, Singapore daisy in Australia,creeping daisy in English,Seruni/Sernai in Indonesia, and Wedeliakuning in Malaysia.

The present research concentrates on development of Sphagneticolatrilobata flower extract coated catheters and determining its anti-biofilm activity. MIC and biofilm assay were determined against major biofilm producing E. coli and S. aureus. Biofilm inhibition was confirmed by Fluorescent microscopic analysis.

\section{MATERIALS AND METHODS}

\section{Collection of Sphagneticolatrilobata flowers and processing}

Sphagneticolatrilobata flowers were collected from the areas of Hope college, Coimbatore (Figure-1). The leaves were washed using distilled water and dried in shade under room temperature. The dried leaves were finely grounded to powders and stored in sterile container for extraction.

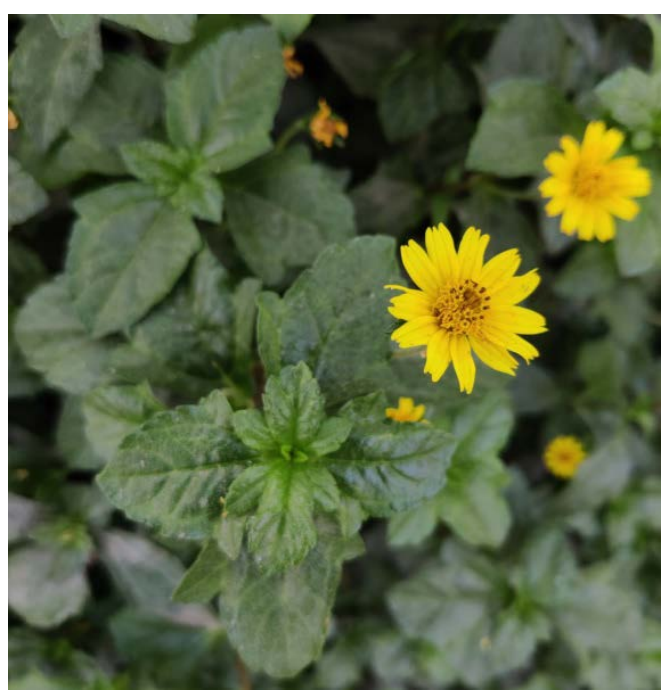

Figure-1: Collection of Sphagneticolatrilobataflowers

\section{Extraction of bioactive compounds using Soxhlet apparatus}

Powders of Sphagneticolatrilobata flowers were placed in a porous bag or "thimble" made from a strong filter paper or cellulose, which is placed, is in thimble chamber of the Soxhlet apparatus. Extraction solvent (methanol) is heated in the bottom flask, vaporizes into the sample thimble, and condenses in the condenser and drip back. When the liquid content reaches 
the siphon arm, the liquid contents is emptied into the bottom flask again and the process is continued. For the study, infusion method of Soxhlet Extraction had been adopted. The dried powder was filled in the thimble and placed in the soxhlet extractor. The extractor had been filled with solvent solution of methanol and the temperature of $60^{\circ} \mathrm{C}$ was set and left for 6hours. The extracts were collected and the solvents were evaporated. The dried extracts were collected and stored in sterile containers

\section{Determining Minimum inhibitory concentration (MIC) of the Sphagneticolatrilobata flower extracts}

Minimum inhibitory concentration (MIC) of the Sphagneticolatrilobata flower extracts was determined against the two major UTI causing pathogen Staphylococcus aureus and Escherichia coli by well diffusion method. Nutrient Agar was prepared and sterilized, and poured into plates. (Nutrient agar Composition (for 100ml): Peptone: 0.5g; Yeast extract: 0.5g, Beef extract: 0.3g, Sodium chloride: 0.5g, Agar $1.5 \mathrm{~g}$; Total pH: $7.0 \pm 0.2$ ). Overnight cultures of test pathogens were cultured and $0.1 \%$ of culture solution of each test organisms was streaked throughout the petri plate with the sterile cotton swab by roating the plate at $60^{\circ}$ angle for each streaking. $6 \mathrm{~mm}$ well borer was used to bore wells on the agar surface of each NA plates. About four concentrations of samples $(2.5 \mathrm{mg} / \mathrm{ml}, 5 \mathrm{mg} / \mathrm{ml}, 7.5 \mathrm{mg} / \mathrm{ml} \& 10 \mathrm{mg} / \mathrm{ml})$ were loaded into the well and the plates were incubated in an incubator at $37^{\circ} \mathrm{C}$ for $48 \mathrm{~h}$. The antibacterial activity was determined in terms of inhibitory zones around the wells loaded with samples in all the Nutrient Agar plates containing test pathogens. The obtained clear zones were observed and measured in millimetre (mm). The minimum concentration showing inhibitory zones was recorded as MIC

\section{Procurement of Urinary catheters and coating of synergistic combination on urinary catheters}

The urinary catheters were procured on Thulasi medicals, Hope college, Coimbatore (Figure-2). The Sphagneticolatrilobata flower extracts was coated using dip and dry method. The extract was prepared and the catheters were dipped for 2mins and left for drying at room temperature. The procedure was repeated twice to achieve the effective coating. Further the coated cathters were used for further analysis. 


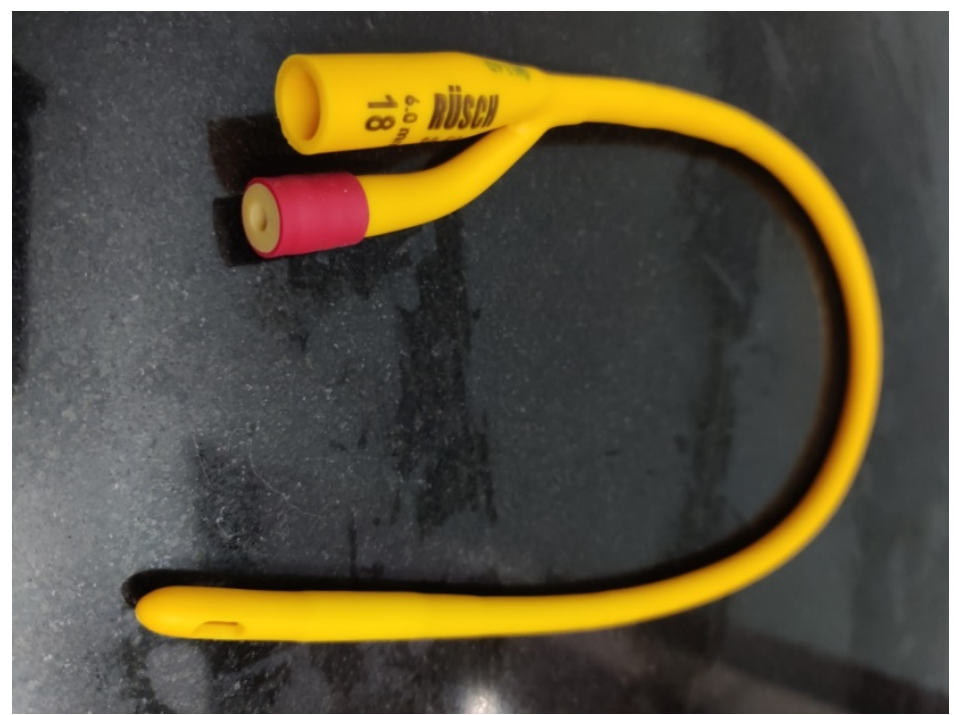

Figure-2: Procurement of urinary catheters

\section{Anti-biofilm activity using Exit-site challenge test}

Exit-site challenge test was used to identify the ability of specific test organism to grow on a type of biomedical materials used in the study. In this method, three-quarter strength of sterile Iso-sensitest semi solid Agar (Thermo scientific) was poured into a sterile boiling tube and allowed to solidify. The surface of the agar was then inoculatedwith $10 \mu \mathrm{l}$ of 18 hours test culture (MRSA). The pre-measured size (length $-15 \mathrm{~mm}$ ) of urinary catheters were cut, sterilized and partially inserted into the Iso-sensitest semi-solid medium through the inoculated area and incubated at $37^{\circ} \mathrm{C}$. Migrating ability of the test bacteria from the exit site down the material track i.e., outside of the materials were assessed visually up to 24-48 hours.

\section{Fluorescent microscopic analysis of biofilm inhibition activity}

The biofilm inhibition ability of flower extracts against Staphylococcus aureus and Escherichia coli visualized by Fluorescents microscopic. Briefly, the glass beads were placed in each well Staphylococcus aureus and Escherichia coli suspensions (50 $\mu \mathrm{L})$ were inoculated into each well along with fresh MFB media and $20 \mu$ l of extract were inoculated and then incubated overnight at $37^{\circ} \mathrm{C}$. Biofilms that formed were then washed twice with PBS to remove any unattached and floating cells and the glass beads were then washed using PBS buffer and stained using crystal violet for Staphylococcus aureus,, stain for Escherichia coli. The stain beads are viewed under microscope. 


\section{RESULTS AND DISCUSSION}

Determining MIC of the Sphagneticolatrilobata Extracts

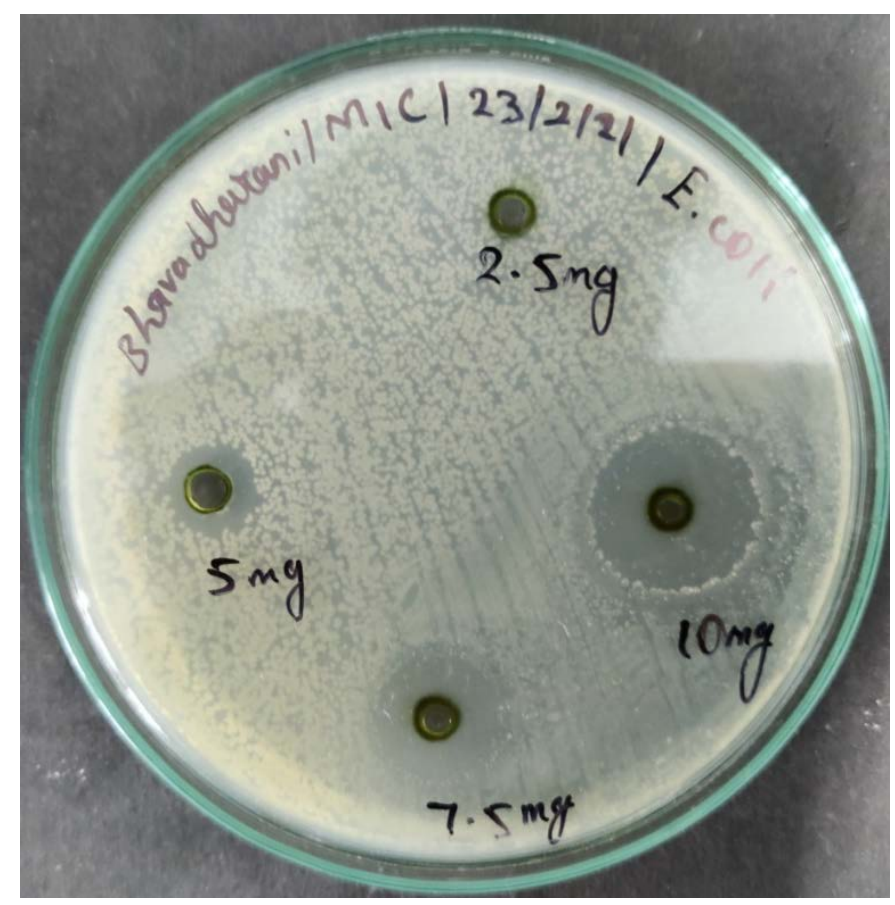

Figure-3: MIC against Escherichia coli

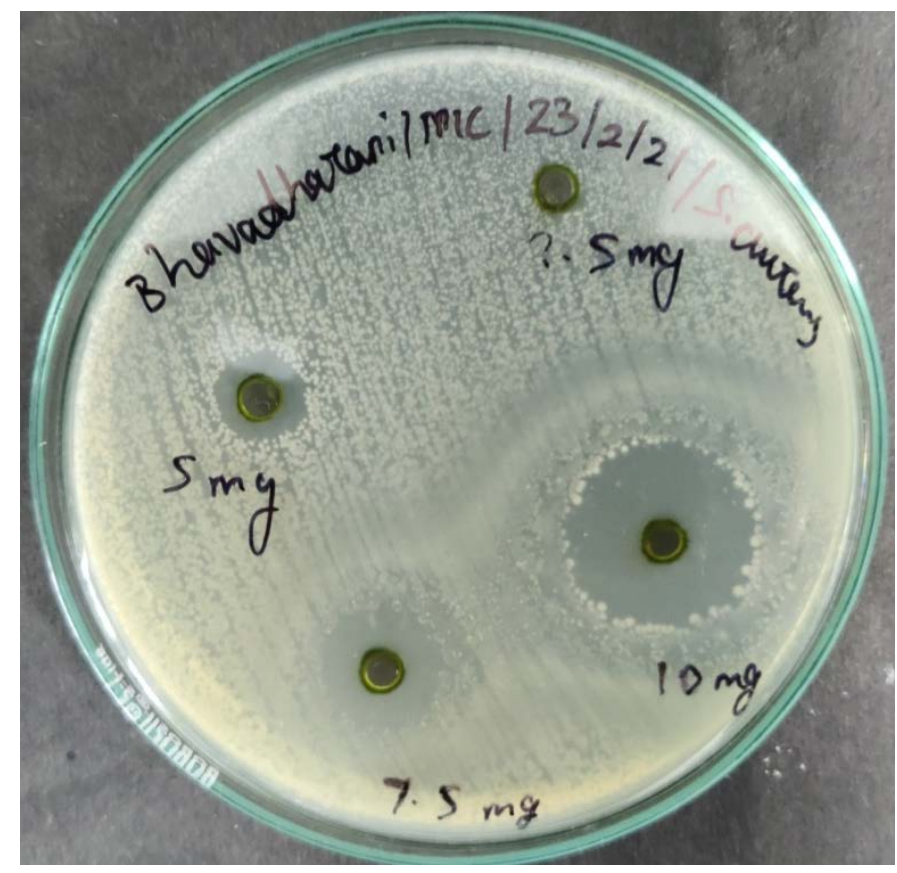

Figure-4: MIC against Staphylococcus aureus 
Table-1: Determining the MIC of the flower extracts

\begin{tabular}{|c|c|c|c|}
\hline \multirow{2}{*}{ S. No } & Concentration (mg/ml) & \multicolumn{2}{|c|}{ Inhibitory zones (mm) } \\
\cline { 3 - 4 } & & Escherichia coli & $\begin{array}{c}\text { Staphylococcus } \\
\text { aureus }\end{array}$ \\
\hline 1 & 2.5 & 0 & 0 \\
\hline 2 & 5 & 8 & 10 \\
\hline 3 & 7.5 & 13 & 14 \\
\hline 4 & 10 & 14 & 17 \\
\hline
\end{tabular}

Minimum inhibitory concentration (MIC) is the lowest concentration required to inhibit the bacteria. $2.5 \mathrm{mg} / \mathrm{ml}$ of flower extracts showed no inhibitory zones (Figure-3 and 4 ). $5 \mathrm{mg} / \mathrm{ml}$ of flower extracts showed slight zone (8mm) against Escherichia coli and $10 \mathrm{~mm}$ against Staphylococcus aureus. $7.5 \mathrm{mg} / \mathrm{ml}$ showed $13 \mathrm{~mm}$ against Escherichia coli and $14 \mathrm{~mm}$ against Staphylococcus aureus. 10mg/ml of flower extracts showed 14mm against Escherichia coli and $17 \mathrm{~mm}$ against Staphylococcus aureus. Therefore, the MIC of the flower extracts against Escherichia coli and Staphylococcus aureus was found to be $5 \mathrm{mg} / \mathrm{ml}$ (Table-1).

\section{Procurement and coating of extracts on catheter}

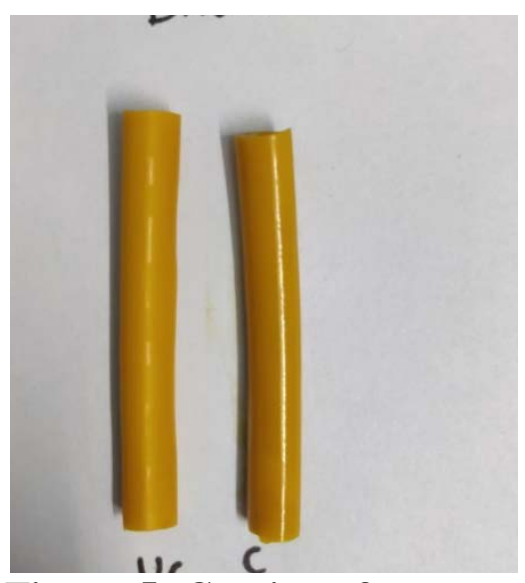

Figure-5: Coating of extracts

Catheters were coated by flower extracts. Dip and dry coating technique was employed. The coated catheters were impregnated flower extracts whereas the uncoated catheters contains no impregnation and remains plain. The coated catheters were shown in Figure-5. 


\section{Anti-biofilm activity of coated catheters}

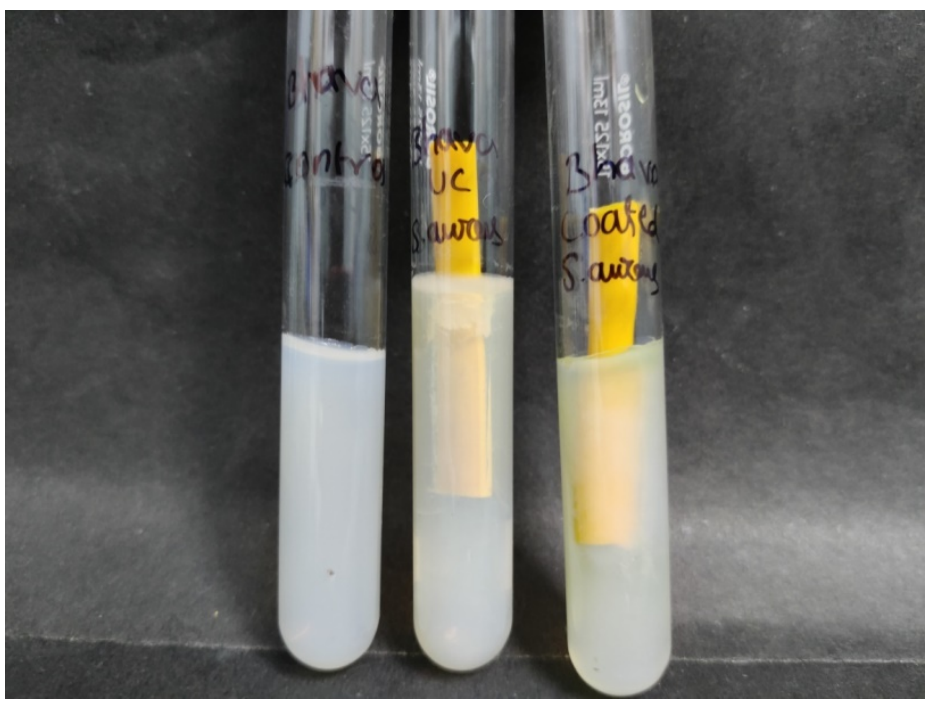

Figure-6: Anti-biofilm activity of coated catheters against $S$. aureus

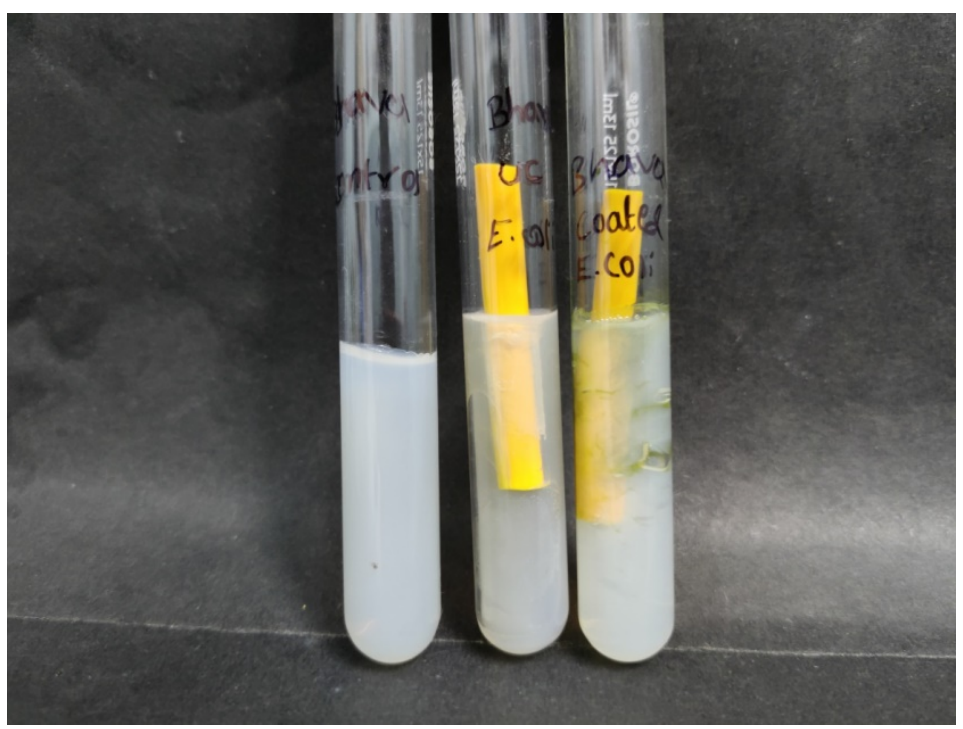

Figure-7: Anti-biofilm activity of coated catheters against $E$. coli

Anti-biofilm activity of the flower extract coated catheters was determined by Exit site challenge test. The coated and uncoated catheters were placed in the agar inoculated with test organism. Formation of biofilm on the surface was observed on uncoated catheters, whereas no biofilm formation was observed on flower extract coated catheters (Figure-6 \& 7). This was confirmed by the Fluorescent microscopic analysis. 
Figure-8: Fluorescent microscopic images

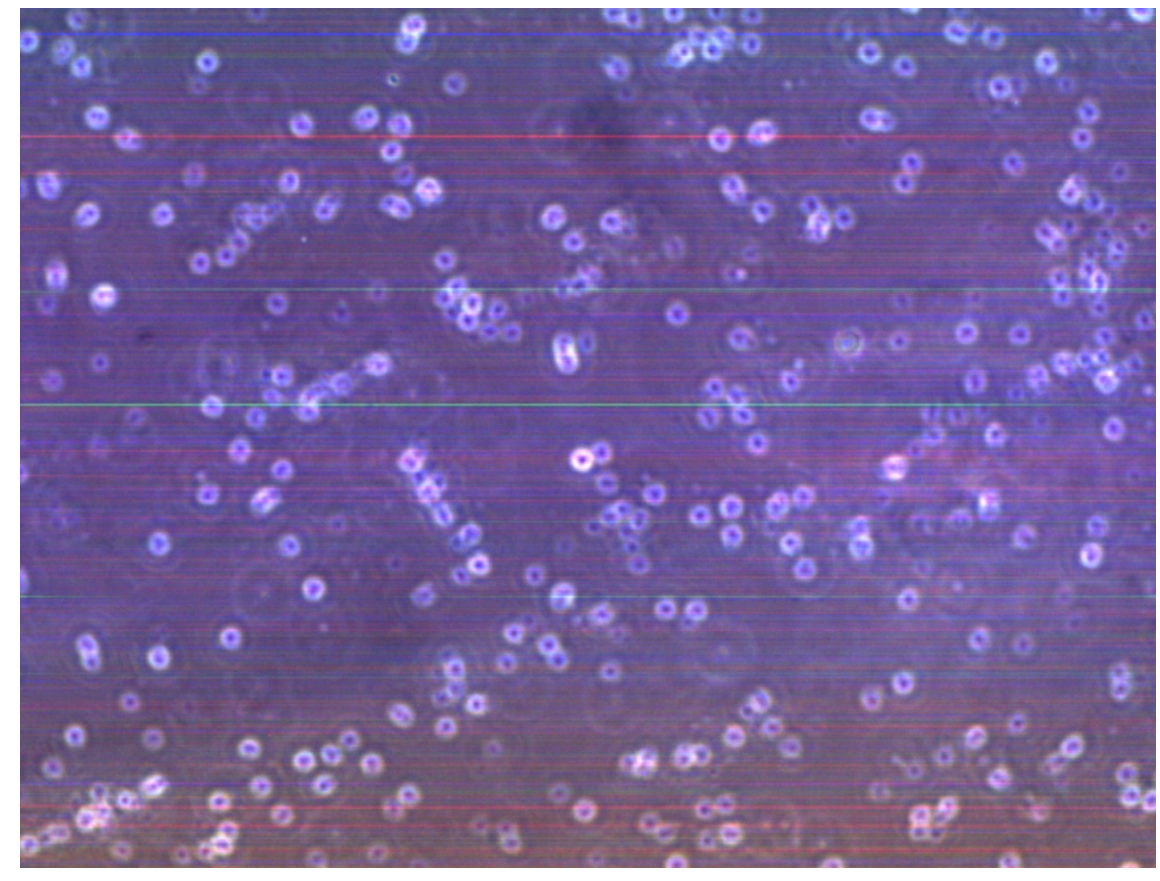

Staphylococcus aureus - control

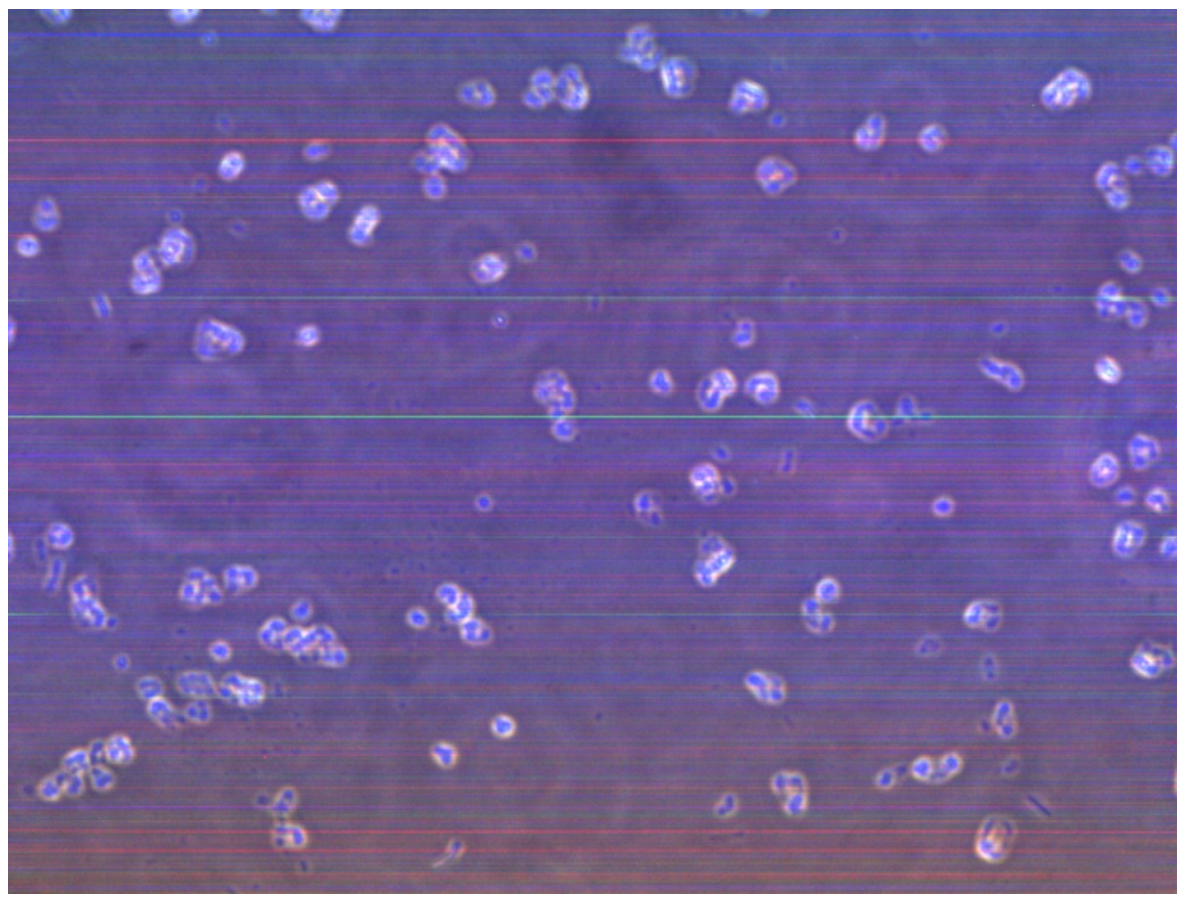

Staphylococcus aureus - extract treated 


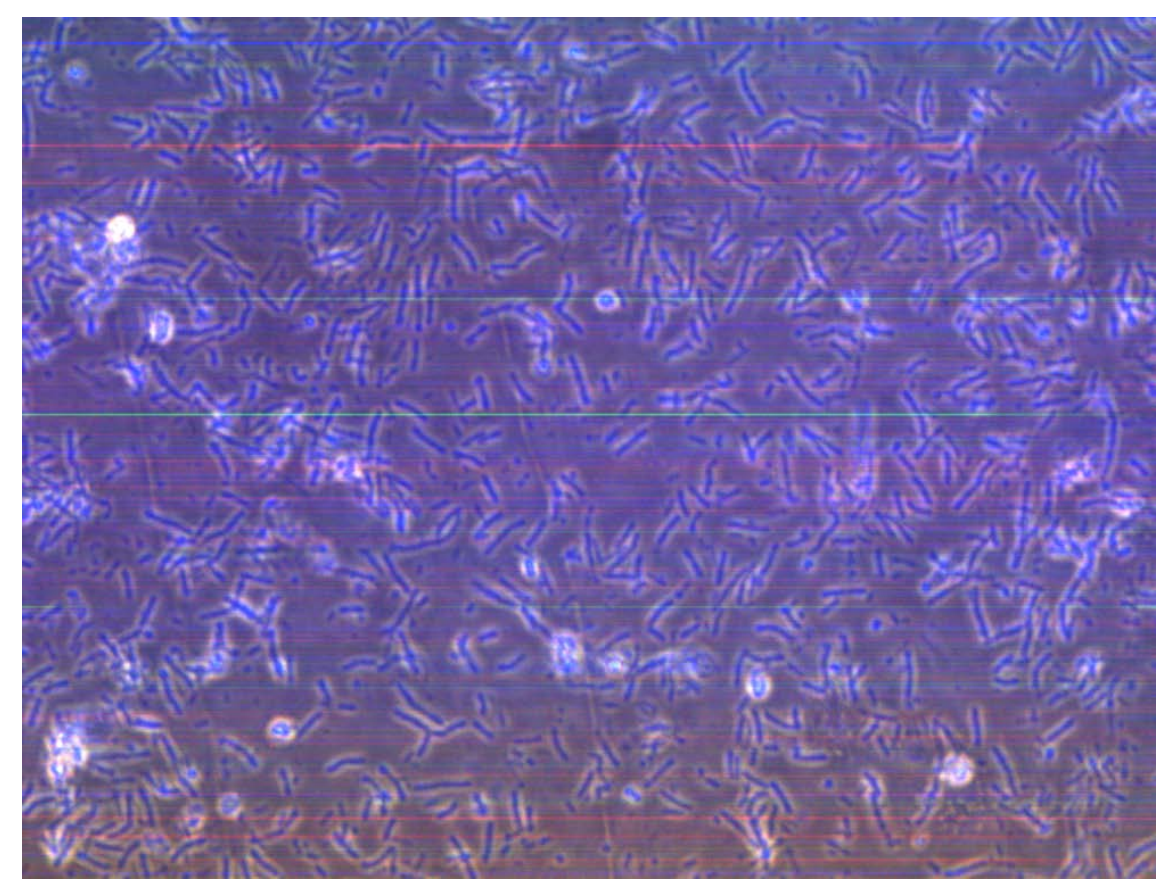

\section{Escherichia coli - Control}

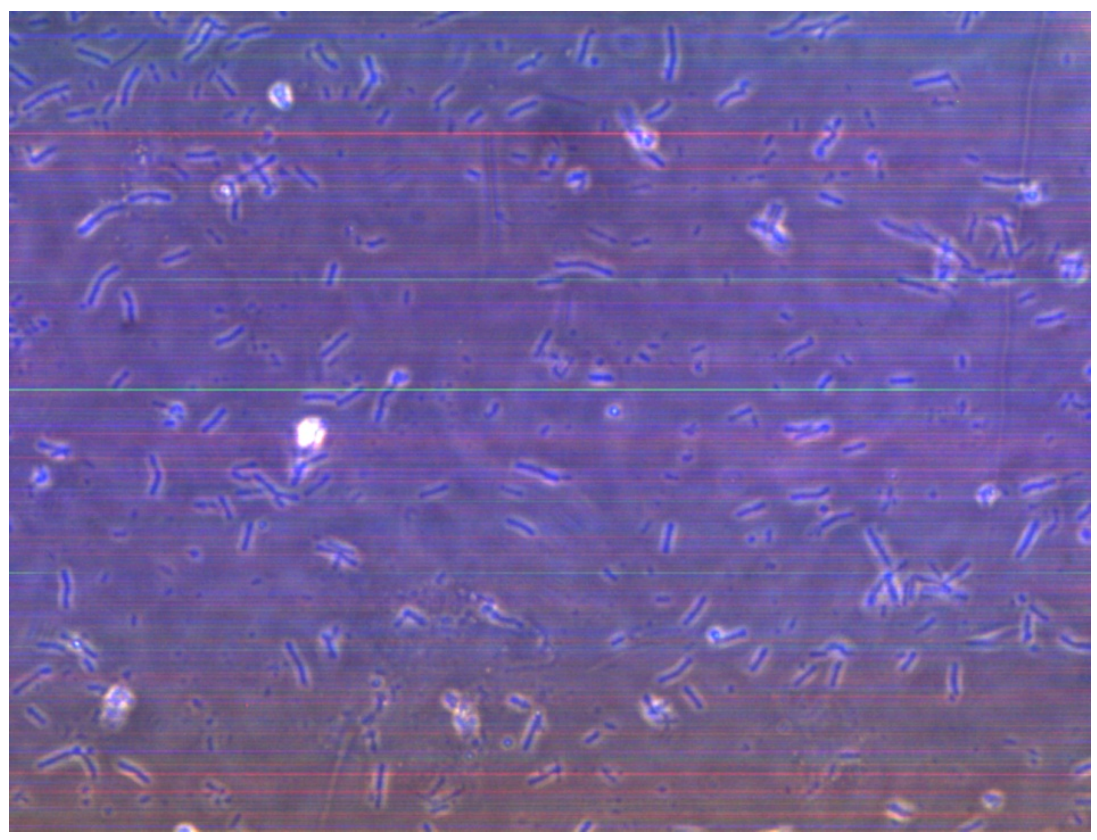

\section{Escherichia coli - extract treated}

Anti-biofilm activity was confirmed by fluorescent microscopy analysis. Biofilm formation was observed on uncoated catheters. Figure-8 shows biofilm formation of $S$. aureus on uncoated catheters. Significant decrease on $S$. aureus was observed on coated catheters.Similarly higher concentration of E. coli was observed on uncoated catheters. Whereas, 
drastic reduction of $E$. coli was observed on flower extract coated catheters. This confirms the biofilm inhibition potential of flower extracts when coated on urinary catheters.

\section{DISCUSSION:}

Plants are the source of medication for preventive, curative, protective or promotive purposes. Medicinal plants are an important source for generating of novel phytomedicine. They provide profound therapeutic benefits, more affordable treatments, effectiveness, less side effects and relatively low cost or less expensive and globally competitive. Using plant derived medicine is also relatively safe compared to synthetic medicines. Many plants have proved to successfully aid in the treatment of ailments including Sphagneticolatriolobata (L.) Pruski(N Buddhakala, et al.,2020).

Some medical microbiologists, when exposed to the theory that bacteria grow preferentially in biofilms on surfaces, felt instinctively that pathogenic organisms must comprise an exception to this rule. In most natural environments, association with a surface in a structure known as a biofilm is the prevailing microbial lifestyle. Surface association is an efficient means of lingering in a favorable microenvironment rather than being swept away by the A biofilm is a population of cells growing on a surface and enclosed in an exopolysaccharide matrix. (HC Flemming, J Wingender , 2010)

Biofilms are notoriously difficult to eradicate and are a source of many recalcitrant infections current. Resistance is an ability of a microorganism to grow in the presence of an elevated level of an antimicrobial. In short, a strain for which the MIC is increased is resistant. By this conventional criterion, biofilm cells do not necessarily show increased resistance. With some exceptions, biofilm cells do not grow better than planktonic cells in the presence of a broad range of antimicrobials(Kim Lewis 2001)

Nosocomial urinary tract infection (UTI) is the most common infection acquired in both hospitals and nursing homes and is usually associated with catheterization. This infection would be even more common but for the use of the closed catheter system. Most modifications have not improved on the closed catheter itself. Even with meticulous care, this system will not prevent bacteriuria. After bacteriuria develops, the ability to limit its complications is minimal. Once a catheter is put in place, the clinician must keep two concepts in mind: keep the catheter system 
closed in order to postpone the onset of bacteriuria, and remove the catheter as soon as possible. If the catheter can be removed before bacteriuria develops, postponement becomes prevention (John W .warren.,2001).

Urinary catheter-related infections are commonly seen in several different patient populations and lead to substantial morbidity. The overall health care costs caused by these infections are sizable given how often urinary catheters are used in acute care settings, extended care facilities, and in persons with injured spinal cords. Recent attention has appropriately focused on biofilm development on the catheter surface because biofilm has important implications for the pathogenesis, treatment, and prevention of catheter-related infection. Because the most important risk factor for infection is duration of catheterization, indwelling urethral catheterization should be avoided or at least limited whenever possible. Additional methods to prevent this infection include aseptic insertion and maintenance use of a closed drainage system, anti-infective catheters in patients at high-risk for infection, and systemic antibiotics in select patients. Alternative urinary collection strategies may be appropriate in certain patient groups. (Saint S, Chenoweth CE. 2003 )

Biofilm-associated bacterial infections frequently caused by S. Epidermidis, S. Aureus, E. Coli, and P. Aeruginosa are found in majority of human diseases. The effectiveness of many antimicrobial drugs has been lost due to the evolution of pathogenic resistance. Thus, microorganisms are no longer susceptible to most of the existing antibiotics and therapeutic agents (Ro“mling and Balsalobre 2012).His study also shows that the Sphagneticolatrilobataextracts are active against UTI causing pathogens. DibyajithLahiriet al., 2019 ,they have listed the various biofilm-forming bacteria and the bioactive compounds being produced from the aerial parts of plants having antibiofilm activity and evaluated them against different biofilm-producing bacterial strains. These studies well correlates with the present study.

\section{CONCLUSION:}

The present study focus on coating of Sphagneticolatrilobata coated catheters for prevention of urinary tract infections (UTI). $7.5 \mathrm{mg} / \mathrm{ml}$ extracts showed $13 \mathrm{~mm}$ against E. Coliand $14 \mathrm{~mm}$ against $S$. Aureus. The extract coated catheter showed significant reduction in biofilms from fluorescent microscopic analysis. Hence Sphagneticolatrilobataflower extract coated catheter can be used to prevent biofilm colonization and urinary tract infections. 


\section{REFERENCES:}

Bazaka K, Jacob MV, Crawford RJ, Ivanova EP. Plasma-assisted surface modification of organic biopolymers to prevent bacterial attachment. ActaBiomaterialia. (2011);7:(2015-2028).

Carneiroet al. CasbaneDiterpene as a Promising Natural Antimicrobial Agent against BiofilmAssociated Infection.Molecules (2011); 16:190-201

DIBYAJIT LAHIRI, SUDIPTA DASH, RACHAYEETA DUTTA and MOUPRIYA NAG, J Biosci (2019) 44:52 volV)

Essawi T, Srour M. Screening of some Palestinian medicinal Plants for antibacterial activity. Journal of Ethnopharm(2000);70:343-349

Fux CA, Costerton JW, Stewart PS, Stoodley P. Survival strategies of infectious biofilms. Trends in Microbiology. (2005);13:34-40.

Ignacio González, Angel Ayuso-Sacido, Annaliesa Anderson, Olga Genilloud, Actinomycetes isolated from lichens: Evaluation of their diversity and detection of biosynthetic gene sequences, FEMS Microbiology Ecology, Volume 54, Issue 3, November( 2005), Pages 401-415.

JL Del Pozo, Robin Patel,Clinical Pharmacology \& Therapeutics 82 (2), 204-209, (2007).

Karlapudi P, Sreenivas, Tirupati, C and Prabhakar..International Journal of Pharmacy and Pharmaceutical sciences,(2012), 4,282.

Kim Lewis Antimicrobial Agents and Chemotherapy Apr (2001), 45 (4) 999-1007; DOI: 10.1128/AAC.45.4.999-1007.(2001)

Liu, C., Jiang, Y., Wang, X. et al. Diversity, Antimicrobial Activity, and Biosynthetic Potential of Cultivable Actinomycetes Associated with Lichen Symbiosis. MicrobEcol74, 570-584 (2017).

Maki DG, Kluger DM, Crnich CJ. The risk of bloodstream infection in adults with different intravascular devices: a systematic review of 200 published prospective studies. Mayo Clinic Proceedings. (2006);132:391-402. 
Mermel LA, Farr BM, Sherertz RJ, Raad II, O Grady N, Harris JS. Guideline for the management of intravascular catheter-related Infectious.Clinical Infectious Disease. (2001);32:1249-1272.

Nicolle, L.E. Catheter-Related Urinary Tract Infection. Drugs Aging 22, 627-639 (2005).

Romling U and Balsalobre C 2012 Biofilm infections, their resilience to therapy and innovative treatment strategies.J.intern. Med. 272 541-561

Sitges-Serra A, Girvent M. Catheter-related bloodstream infections.World Journal of Surgery. (1999);23:589-595.

Tamilvanan S, Venkateshan N, Ludwig A. The potential of lipid-and polymer-based drug delivery carriers for eradicating Biofilm consortia on device-related nosocomial infections.J Control Release. (2008);128:2-22

ViviMardina, SyafruddinIlyas, [...], and MasittaTanjung,JAdv Pharm Technol Res. (2020) JulSep; 11(3): 123-127.

Wei Wu, Ren-Chao Zhou, Guang-Yan Ni, HaoShen, Xue-Jun Ge,Biological invasions 15 (1), 75-88, (2011) 